\title{
BETA FUNCTIONAL CONNECTIVITY MODULATION DURING THE MAINTENANCE OF MOTION INFORMATION IN WORKING MEMORY: IMPORTANCE OF THE FAMILIARITY OF THE VISUAL CONTEXT
}

\author{
C. CALMELS, a* M. FOUTREN ${ }^{a, b}$ AND C. J. STAM ${ }^{c}$ \\ INSEP, Institut National du Sport, de \\ l'Expertise et de la Performance, Paris, France \\ Universite' Paris-Est Marne la Valle'e \\ France \\ Department of Clinical Neurophysiology, VU University \\ Medical Centre, Amsterdam, The Netherlands
}

\begin{abstract}
The purpose of this study was to examine whether mechanisms, involved during the maintenance of familiar movement information in memory, were influenced by the degree of familiarity of the display in which the movements were embedded. Twelve gymnasts who possessed high visual and motor familiarity with the movements employed in this study, were recruited. They were invited to retain for a short period of time familiar movements viewed previously and presented under different displays with the aim of recognizing them at a later stage. The first display was a realistic, familiar display which presented videos of movements. The second display was an unfamiliar impoverished display never experienced in every day life which showed point-light movements. Activity during the maintenance period was considered in five frequency bands $(4-8 \mathrm{~Hz}, 8-10 \mathrm{~Hz}, 10-13 \mathrm{~Hz}, 13-20 \mathrm{~Hz}$, $20-30 \mathrm{~Hz}$ ) using a non-linear measure of functional connectivity. The results in the $13-20 \mathrm{~Hz}$ frequency band showed that functional connectivity was greater within the frontal and right temporal areas during the unfamiliar display (i.e., point-light maintenance condition) compared to the familiar display (i.e., video maintenance condition). Differences in functional connectivity between the two maintenance conditions in the beta frequency band are mainly discussed in the light of the process of anticipation. Subjects' perception of the expected difficulty of the upcoming recognition task is discussed. 2012 IBRO. Published by Elsevier Ltd. All rights reserved.
\end{abstract}

Key words: working memory maintenance, biological movement, functional connectivity, display familiarity.

\footnotetext{
*Corresponding author. Address: Mission Recherche, Institut National du Sport de l'Expertise et de la Performance, 11 Avenue du Tremblay, 75012 Paris, France. Tel: +33-(1)-41-74-43-73; fax: +33-(1)-41-74-45-35.
}

0306-4522/12 \$36.00 2012 IBRO. Published by Elsevier Ltd. All rights reserved. http://dx.doi.org/10.1016/j.neuroscience.2012.03.045

E-mail address: claire.calmels@insep.fr (C. Calmels).

Abbreviations: EEG, electroencephalography; $\mathrm{fMRI}$, functional magnetic resonance imaging; MEG, magnetoencephalography; PLI, phase lag index; SL, synchronization likelihood.

\author{
INTRODUCTION
}

In everyday life, humans have to actively hold information active in memory for short periods of time in order to subsequently perform motor or cognitive tasks successfully.

Mechanisms underlying the active maintenance of information in working memory have been extensively investigated in the scientific literature and many research groups have used the multiple-component model of working memory developed by Baddeley and Hitch (1974) and Baddeley $(1986,2000,2010)$ to direct their studies and support and explain findings. To examine these mechanisms, different techniques including functional magnetic resonance imaging (fMRI; Postle et al., 2000; Pochon et al., 2001; Sakai et al., 2002; Fiehler et al., 2008) or electroencephalography/magnetoencephalography (EEG; Sarthein et al., 1998; Tallon-Baudry et al., 1998, 1999; Stam et al., 2002; Hwang et al., 2005; Sauseng et al., 2005; MEG; Altamura et al., 2010) and different paradigms such as recall or Sternberg tasks (recall tasks; Sarthein et al., 1998; Stam et al., 2002; Sternberg tasks; Tallon-Baudry et al., 1998, 1999; Hwang et al., 2005; Sauseng et al., 2005; Altamura et al., 2010) have been employed. In all the aforementioned studies, the stimuli used only included still images (e.g., letters, digits, words, smooth shapes, pictures, matrixes containing coloured targets, and spatial locations). Dynamic displays, such as biological movements, have scarcely been employed in working memory studies.

To our knowledge, two studies have presented hand/ finger movements to examine the mechanisms to hold movement information active in memory after a kinaesthetic stimulus presentation (Fiehler et al., 2008) and a visual stimulus presentation (Calmels et al., 2011). Using fMRI, Fiehler et al. (2008) examined the cortical regions involved when subjects kept in memory for short periods of time hand movement information in order to accomplish a recognition task at a later stage. In their study, movement information was exclusively kinaesthetic and visual input was systematically suppressed. Subjects were blindfolded and movement recognition was completed on a kinaesthetic basis. Results revealed that anterior intraparietal sulcus and adjacent areas were involved in the maintenance of kinaesthetic information. Calmels et al. (2011)

49

investigated, via EEG, the functional connectivity among professional pianists and musically naı"ve subjects who were instructed to remember sequential finger movements viewed previously with the aim of either replicating them or recognizing them subsequently. The results showed that in the beta 
frequency band and in musically naı"ve subjects, functional connectivity was greater within the occipital, parietal, central, frontal, right and left temporal areas when the subjects were invited to remember the observed movement in order to replicate it compared to the recognition condition in which they had to recognize it. In addition, under the condition for replica, functional connectivity in professional pianists was weaker in the central area compared to musically naı" ve subjects.

It would be of interest to consider the mechanisms of movement information maintenance in working memory after the presentation of visual dynamic stimuli in a Sternberg task. More specifically, the maintenance mechanisms enrolled in response to different visual stimuli depicting highly familiar biological movements warrant further attention since this topic has not been addressed in the literature. In the current study, different visual stimuli depicting familiar biological movements were presented. These stimuli were different with respect to their degree of familiarity. The first kind of visual stimulus was presented via videos of "real" movements and emanated from a realistic, ecological display. It contained large amounts of structural and contextual information (i.e., form, shape, colour, texture). The second one was devoid of this type of information and was displayed using the technique of point-light displays pioneered by Johansson (1973). This display was an unfamiliar impoverished display never experienced in every day life. Subjects were selected from among a population of expert female gymnasts who were visually and motorically highly familiarized with the movements employed in the experimental procedure. This recruitment procedure guaranteed that the factor which has been manipulated in the present study was the display (unfamiliar vs familiar). The investigation of cortical mechanisms, in particular levels of synchronization between different brain areas, was performed using the phase lag index (PLI). PLI is a marker of functional connectivity which is invariant against the presence of common sources (volume conduction and active reference electrodes) (Stam et al., 2007).

It was hypothesized that maintenance of familiar movement information in working memory was influenced by the degree of familiarity of the display in which this movement was embedded. Mechanisms to maintain temporarily in memory motion information presented via point-light displays may require a greater treatment cost, reflected by a greater functional connectivity, in comparison to motion information presented via videos of "real" motions. Extra processing may be required to process point-light displays since individuals are not used to experiencing these artificial and impoverished displays in the daily life. Functional connectivity differences are expected to occur in the beta frequency band since beta oscillations are recognized as being linked to the retention of information (Tallon-Baudry et al., 1998, 1999, 2001; Hwang et al., 2005; Leiberg et al., 2006; Pesonen et al., 2007; Altamura et al., 2010; Calmels et al., 2011). More specifically, beta activity is related to the activity of subvocal rehearsal during the maintenance of information in the working memory (Hwang et al., 2005) but also to the process of (mental) imagery (Tallon-Baudry et al., 1998, 1999, 2001). It has also been demonstrated that beta rhythm is at play during imagery of motor activity. It is desynchronized over the sensorimotor cortical areas (Pfurstcheller and Neuper, 1997; Pfurtcheller et al., 1997; Neuper and Pfurtscheller, 1999; McFarland et al., 2000).

\section{METHOD}

Subjects

Fifteen French female expert gymnasts, who had normal vision and no past neurological or psychiatric history, participated voluntarily in the study. Subjects were not carrying any injury at the time of the study. Information on the aims of the investigation was not provided. To guarantee that the subjects possessed motor and visual familiarity of the movements employed in the study, they were invited to evaluate on a Likertscale, graded from 0 to 10 (" 0 " never and " 10 " very often), how often they saw the movements and how often they performed the movements. Gymnasts who scored below " 8 " were discarded from the study. After assessment using the Edinburgh Handedness Inventory (Oldfield, 1971), all subjects were classed as right handed. Participants gave their written informed consent. For subjects who were under the age of 18 , parental or guardian consent was also obtained. Approval for the study was obtained by the local ethics committee (Comite' de Protection des Personnes d'lle de France VI, CPP and the Agence

Franc, aise de $\mathrm{Se}^{\prime}$ curite' Sanitaire des Produits de Sante', AFSSAPS, ID RCB: 2009-A00934-53).

Three subjects were additionally discarded from the study due to high electrode impedance values and noisy EEG waveforms. Thus, data from the twelve remaining subjects ( mean age $=21, \mathrm{SD}=4.1$ ) were considered.

\section{Task and production of videos}

An international female gymnast performed 30 direct series of four acrobatic movements. This gymnast did not participate in the present study. The movements were performed with or without flight phase in the forward, sideward, or backward movement. The series were matched according to difficulty and were selected among a panel of 80 possible direct connections of four acrobatic movements. Connections considered as easy to remember (i.e., score assessment of 1,2 , or 3 on a 5 pointLikert scale by two national standard judges) were excluded. 
The international gymnast was filmed in a gymnasium on the floor area performing the 30 direct connections. The gymnast performed all the connections at a similar angle relative to the camera. In the first case, a digital camera was used to record 30 ten second-colour videos. In the second case, 10s point-light displays were generated for the same series using the optoelectronic Vicon 612 system. Eight infrared cameras (Charge Coupled Device) registered the spatiotemporal positions of 32 retroreflective markers at a sampling rate of 120 $\mathrm{Hz}$. These were located at the conventional standardized marker computation of functional connectivity via the PLI was more elaborated compared to the computation employed in the former paper (i.e., synchronization likelihood, SL). Indeed, PLI is less sensitive to the influence of common sources (volume conduction and/or active reference electrodes) than SL (Stam et al., 2007). To sum up, the investigated mechanisms, areas of interest, frequency bands, andcalculationoffunctionalconnectivity were different between the two studies.

\section{EEG recording}

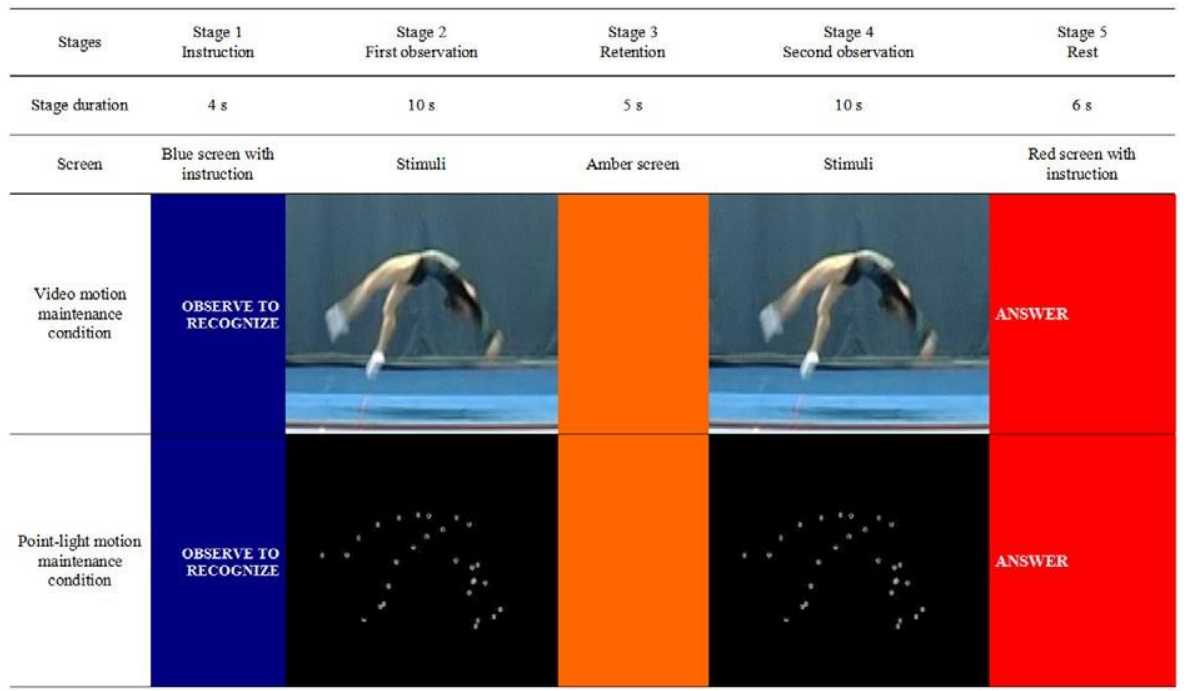

Fig. 1. Schema for one trial according to maintenance conditions used by Hars et al. (2011).

set (Plug-In-Gait markers, Vicon Motion Systems). Two categories of stimuli were obtained. The first one took into account the colour, shape, shading, and contours of the acrobatic movements (i.e., video motion) while the second was greatly simplified and characterized the movements by dots of light (i.e., point-light motion) (see Fig. 1). The point-lights were displayed in a white against black background.

\section{EXPERIMENTAL PROCEDURE}

All experiments conformed to a named local as well as International Guidelines on the ethical use of animals and that all efforts were made to minimise the number of animals used and their suffering. The experimental procedure comprised two parts: an EEG recording and an interview. The first part of the experimental procedure in the present study has been described in a previous paper (Hars et al., 2011). More accurately, the current paper reports additional analysis of EEG data collected previously. In the earlier paper, the authors examined the cortical mechanisms during observation whereas in the present paper, mechanisms after the observation, namely during the retention stage, were investigated. Because observationandretentionaretwodifferentprocesseswhichinvolve differentcortical areas andoscillations, theareas ofinterest andthe frequency bands examined in the present and the former study are different. Motor related areas, temporal and occipito-parietal areas were considered in the earlier study whereas frontal, temporal, central, parietal, and occipital areas were investigated in the present paper. Theta and alpha frequency bands were considered in the former study and beta was investigated in the current paper. Moreover, here,
During the EEG recording, the subjects sat in a darkened room with their pronated forearms lying on armrests. They were examined in three conditions: (i) a control condition, (ii) a video motion maintenance condition, and (iii) a point-light motion maintenance condition. EEG measures were recorded during all three conditions.

Control condition. The control condition was always presented first and each visualization lasted 60s. In this condition, the subjects observed passively an amber screen.

Video motion maintenance condition. The video motion maintenance condition comprised 30 individual trials each lasting 35s. Each trial included five stages projected via a video display. Three screen colours (blue, amber, and red $^{1}$ ) helped the subject follow the procedure (see Fig. 1). In stage 1 (blue screen, duration $4 \mathrm{~s}$ ), the subject was instructed to observe a series of four acrobatic movements with the aim of recognizing them subsequently. In stage 2 (duration 10s), the subject viewed the video motion of the series of four acrobatic movements (i.e., cue stimulus). After the video motion observation, the subject was asked to stay focused for 5 s (stage 3 , amber screen) before completing the recognition task. In stage 4 (duration 10s), a second video motion of a series of four acrobatic movements (i.e., probe stimulus) was presented and the subject had to decide whether this series was similar or dissimilar to the one viewed in stage 2 (i.e., cue stimulus). Two series were defined as dissimilar when there was one different acrobatic movement between the two series. This difference occurred either in the middle or at the end of the series. A total of $50 \%$ of the series were similar. Clenching or not clenching of the fist by the subject was used to

${ }^{1}$ For interpretation of colour in Fig. 1, the reader is referred to the web version of this article. 
indicate the response in stage 5 (red screen, 6s). In this final stage, the subject was encouraged to relax and to blink the eyes if necessary.

Point-light motion maintenance condition. In the point-light motion maintenance condition, the 30 trials were performed in a similar manner to those used in the video motion maintenance condition. In contrast, stages 2 and 4 were modified. While the subjects observed the same set of series of four acrobatic movements displayed in the video motion maintenance condition, the display under the point-light motion maintenance condition was impoverished and characterized by dots of light (see Fig. 1). As previously mentioned, $50 \%$ of the series were similar.

A photoresistive diode that responded to the screen colour change was used to synchronize the EEG signal and the videos. Before providing the subjects with the instructions related to the experiment, the control condition was presented to them. This was followed by an explanation of the experiment and a 10 min-period during which the subjects had an opportunity to familiarize themselves with the tasks to be performed. The 60 trials ( 30 trials for the video motion maintenance condition and 30 trials for the point-light motion maintenance condition) were randomized and distributed at random in four $8 \mathrm{~min} 45 \mathrm{~s}$ blocks. Each block was thus composed of 15 trials stemming from the two maintenance conditions and separated by a five minute rest period. The experimenter monitored the correctness of the answers provided in the two maintenance conditions for recognition. Incorrect answers were discarded.

\section{Interview}

After the EEG recordings, interviews (Vermersch, 2003) were conducted to provide an occasion to study a combination of the subjective experience of the subject with more objective results emanating from scientific analyses. The interviews lasted from 10 to $15 \mathrm{~min}$. The subjects were invited to identify the strategies they had employed during the retention phase (i.e., stage 3 ). They were also asked to assess, via a $10-$ point Likert scale

(" 0 " very difficult and " 10 " very easy), the difficulty to maintain in memory, and recognize movements under a point-light and video motion maintenance conditions.

\section{Data acquisition and recording}

Electrical brain activity was recorded using 64 electrodes mounted in an elastic lycra cap (SynAmps2 64 channels QuickCap, Neuromedical supplies, Charlotte, NC, USA) and placed in accordance with the international 10-10 system (Nuwer et al., 1999). Mastoids were used for the reference electrodes and the ground electrode was located between FPZ and FZ. In addition, electro-oculograms (EOG) were registered from the canthi of both eyes (horizontal EOG) and the supra and infra orbital of the left eye (vertical EOG). Throughout the experimentation, electrode impedance was kept homogenously below $5 \mathrm{kX}$. Amplifier bandwidth was set between 0.05 and $100 \mathrm{~Hz}$. While the initial sampling frequency was $500 \mathrm{~Hz}$, data were downsampled to $250 \mathrm{~Hz}$ for further analysis. $A D$ resolution was $24 \mathrm{bit}$.

\section{Functional connectivity: PLI}

Functional connectivity was assessed using the PLI (Stam et al., 2007). Here, we followed the description provided in Douw et al. (2010, p. 111): The PLI calculates synchronization between time series by reflecting the consistency with which one signal is phase leading or lagging with respect to another signal. The PLI exploits the asymmetry of the distribution of instantaneous phase differences between two signals. It assumes that the presence of a consistent, nonzero phase lag between two time series cannot be explained by volume conduction alone. Thus, finding true interactions instead of volume conduction effects is more likely when using this method. The PLI ranges between 0 and 1 , and a PLI of more than 0 indicates phase locking to a certain extent, whereas a PLI of 0 indicates no coupling or coupling with a phase difference centred around $0 \pm p$ radians. An index of the asymmetry of the phase difference distribution can be obtained from a time series of phase differences $\mathrm{DU}\left(\mathrm{t}_{\mathrm{k}}\right), \mathrm{k}=1, \ldots, \mathrm{N}$ in the following way:

\section{PLI 1/4 jhsign $1 / 2 \mathrm{D} / \mathrm{dt}_{\mathrm{k}} \mathrm{Pij}$}

$\begin{array}{lllll}\text { See Stam et } & \text { al. } & \text { (2007) for } & \text { a } \\ \text { complete description } & \text { of } & \text { PLI calculation. }\end{array}$

Data processing

EEG data were analysed in five frequency bands: $4-8 \mathrm{~Hz}, 8-10 \mathrm{~Hz}, 10$ $13 \mathrm{~Hz}, 13-20 \mathrm{~Hz}$, and $20-30 \mathrm{~Hz}$, in the third stage of the two maintenance conditions (i.e., retention stage: 14-19s), and in the control condition. Data processing consisted of five steps. First, in the case of channels from which the data were evaluated as unexpectedly corrupted by noise, those channels were reconstructed as a linear combination of their nearest neighbours (Scan 4.4 software, Revision E, 2007). Second, EEG data were reformatted in a common average montage. Stam and de Bruin (2004) have shown that montages using mastoids as a reference accentuated long-distance coupling at the cost of small-scale detail, whereas source montages displayed the reverse. Common average montages had intermediate properties (Nunez et al., 1992) and such a montage was used in the present study. Third, ocular (blink) artefacts were reduced via spatial filtering (Scan 4.4 software, Revision E, 2007). Fourth, the EEG trials were segmented. For the two maintenance conditions, only the third stage (14-19s) was considered for the EEG analysis. This stage was reduced to $4 \mathrm{~s}$ in length and the first second was discarded from further analysis to allow the subject to get accustomed to the change of the colour screen. For the control condition, lasting 60s, the 15 first seconds were discarded and the following four seconds were selected for analysis (15-19s). These time points were selected to coincide with the time window of the retention stage under the video and point-light maintenance conditions used for analysis. Finally, PLI was computed for all the electrode pairs, for the last four seconds of the third stage of each of the trials in the two maintenance conditions, for each subject, and frequency band. The PLI values were then averaged across trials for each subject, maintenance condition, electrode, and frequency band. For the 4s-period of the control condition, the same procedure was adopted. To control for variability in PLI values due to individual differences such as scalp thickness, electrode placement, and impedance, functional connectivity was computed as the ratio of the PLI value during the two maintenance conditions relative to the PLI value during the control condition

(i.e., PLlfinalPL = PLlpoint-light condition/PLIcontrol condition and PLlfinalvideo = PLlvideo condition/PLIcontrol condition) (Ulloa and Pineda, 2007; Pineda and Hecht, 2009). A ratio of more than 1 indicates that the PLI value under the experimental condition is greater than the value under the control condition or vice versa. Conversely, a ratio equals to 1 means that the PLI value under the experimental condition is the same as the value obtained under the control condition. This ratio procedure was also undertaken to remove connectivity occurring during the experimental conditions and which was not related to the task to be performed.

\section{Statistical analysis}

To reduce the degrees of freedom in the statistical analyses, PLI from neighbouring electrode sites were averaged together to obtain one overall PLI value for each of the six following areas: frontal area (F7-F5F3-F1-FZ-F2-F4-F6-F8), left temporal area (FT7-T7-C5-TP7-P7), central area (FC5-FC3-FC1- $\mathrm{FCZ}-\mathrm{FC} 2-\mathrm{FC} 4-\mathrm{FC} 6-\mathrm{C} 3-\mathrm{C} 1-\mathrm{CZ}-\mathrm{C2}-\mathrm{C} 4)$, right temporal area (FT8-T8-C6-TP8-P8), parietal area (CP5-CP3-CP1CPZ- CP2-CP4-CP6-P5-P3-P1-PZ-P2-P4-P6), and occipital area (PO7$\mathrm{PO} 5-\mathrm{PO} 3-\mathrm{POZ}-\mathrm{PO} 4-\mathrm{PO}-\mathrm{PO}-\mathrm{O} 1-\mathrm{OZ}-\mathrm{O} 2)$ (see 
Fig. 2).

All statistical analyses were performed using Statistica 7.1. First, for each of the six frequency bands, 2 (conditions) 6 (areas)repeatedmeasuresANOVAs were performed.Therewere two within-subject factors; Condition (two levels: point-light motion maintenance and video motion maintenance) and Area (six levels: frontal, left temporal, central, right temporal, parietal, and occipital). Post hoc comparisons were calculated using Duncan tests where ANOVA results were significant and effect sizes (ES) for repeated measures (Long and Van Stavel, 1995) were reported.

When the interaction conditions by areas was significant, post hoc Duncan tests' comparisons were performed between

PLlfinalPL and PLI finalvideo values for each area to determine whether PLlfinal values under the point-light condition were different from those obtained under the video condition. Duncan tests' comparisons were also calculated between the $\mathrm{PL}_{\text {final }}$ values of each area (e.g., frontal area $\mathrm{PL}_{\text {final }}$ value vs left temporal area $\mathrm{PL}$ ffinal $_{\text {value, frontal area }} \mathrm{PL} \mathrm{f}_{\text {final }}$ value vs central area $\mathrm{PL}_{\text {final }}$ value, frontal area $\mathrm{PL}_{\text {final }}$ value vs right temporal $\mathrm{PL}_{\text {final }}$ value, frontal area $\mathrm{PLI}_{\text {final }}$ value vs parietal area $\mathrm{PLI}$ final value, frontal area $\mathrm{PL}_{\text {final }}$ value vs occipital area $\mathrm{PL} \mathrm{I}_{\text {final }}$ value, left temporal area $\mathrm{PL}_{\text {final }}$ value vs central area PLIfinal value, etc.). These comparisons allowed analysis of the functional connectivity between areas. Areas were thus grouped according to the value of their PLIfinal and to statistically significant results. This procedure, previously employed by Cochin et al. (1998) and Calmels et al. (2006), allows the classification of areas into groups for each considered condition. Further details are provided in the Results section.

Before the ANOVA computation, the normality of the data was checked with the Kolmogorov Smirnov test. To verify the sphericity assumption in repeated measures designs, Mauchley's sphericity test was used.

Second, Wald-Wolfowitz runs tests were conducted. This analysis was performed when the interaction conditions by areas was significant. It verified whether the ratios under each maintenance condition (i.e., PLlfinalPL = PLIpoint-light condition/

$\mathrm{PLI}$ control condition and PLIfinalVideo $=\mathrm{PL}$ lvideo condition $/ \mathrm{PLI}_{\text {control condition }}$ ) and within each of the six areas of interest were statistically different from a theoretical ratio of 1 . If this was the case, it meant that PLI values under the maintenance conditions were significantly different to the PLI value under the control condition. As recommended by Siegel (1956), an adjusted-P value was adopted because of the small sample size. To address the problem of multiple comparisons, significant levels for the Wald-Wolfowitz runs tests were adjusted providing an alpha level of $P$ $<.00417$ since 12 comparisons were examined within each of the six areas and for the two maintenance conditions.

\section{RESULTS}

Qualitative results

Regardless of condition, three (memory) strategies were identified during the interviews as being used by the subjects during the retention stage. The first was subvocal rehearsal and was employed by seven and six subjects respectively under the point-light and video motion maintenance conditions. The second was an

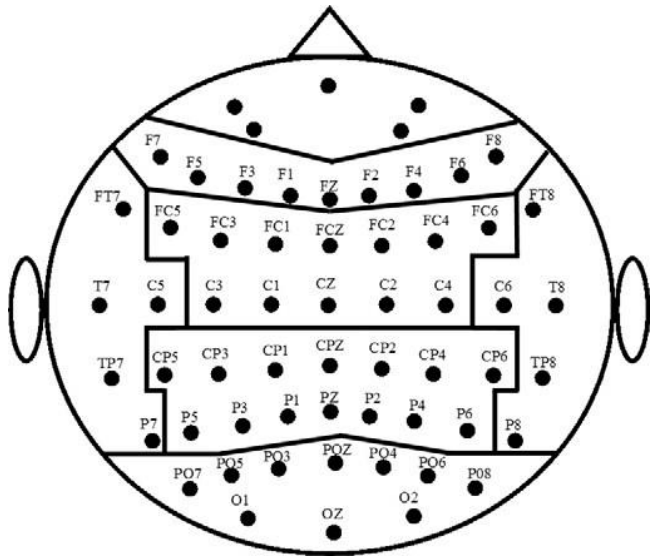

association of subvocal rehearsal and mental imagery. This association was used by four subjects under the point-light motion maintenance condition whereas five subjects used it under the video motion maintenance condition. Finally, two subjects used no strategies (i.e., one subject under the pointlight motion maintenance condition and the second subject under the video motion maintenance condition) (see Table 1). The subjects also perceived the point-light motion maintenance condition to be more difficult and felt it required more concentration than the video motion maintenance condition.

\section{Behavioural results}

During the point-light motion and video motion maintenance conditions, the percentages of correct answers performed by the subjects were, respectively, $92.50 \%(S D=6.98)$ and $98.06 \%$ $(\mathrm{SD}=3.00)$. This difference was statistically significant (Wilcoxon, $\mathrm{T}=5.50, \mathrm{P}=.04$ ). The subjects also reported that the maintenance of movement information from a video display was easier compared to that of the same movements from a pointlight display (8.36 vs 6.36 , Wilcoxon, $\mathrm{T}=0.00, \mathrm{P}=$ .003346).

\section{EEG/PLI results}

2 (Conditions) 6 (areas) repeated-measures ANOVAs were computed. The EEG data were normally distributed and no sphericity violations were observed. Only results for conditions by areas were reported in detail since they were directly linked to the goal of the present study. A significant conditions areas interaction was only revealed in the retention stage for the 13$20 \mathrm{~Hz}$ frequency band, $\mathrm{F}(5,55)=2.624, \mathrm{P}<.035$ (see Table 2 ).

Wald-Wolfowitz runs tests with Bonferroni correction revealed significant differences between the ratios for the two maintenance conditions and a theoretical ratio of 1 within each of the six areas and $\left(\mathrm{Z}_{\mathrm{adj}}=3.9655, \mathrm{P}_{\mathrm{adj}}=.000073,3\right.$ runs $)$. $\mathrm{PLI}$ values under the maintenance conditions were significantly different to PLI values under the control condition. More specifically, PLI values under the point-light motion maintenance condition were higher than PLI values under the control condition within

Fig. 2. The areas of interest.

all areas except within the left temporal area. PLI values under the video motion maintenance condition were weaker compared to $\mathrm{PLI}$ values under the control condition within the frontal, right temporal, and occipital areas.

Duncan post hoc tests were computed and two results obtained. First, significant PLI differences were detected between the two maintenance conditions for the frontal and right temporal areas in the $13-20 \mathrm{~Hz}$ frequency band. PLlfinal values under the 
point-light motion maintenance condition were larger than $\mathrm{PLI}_{\text {final }}$ values under the video motion maintenance condition within the frontal and right temporal areas $(1.01072$ vs $0.98178, \mathrm{P}<.05, \mathrm{ES}=.23$, frontal area; 1.02180 vs $0.99221, \mathrm{P}<.05, \mathrm{ES}=.16$, right temporal area) (see Fig. 3).

Second, in the $13-20 \mathrm{~Hz}$ frequency band, Duncan post hoc tests performed on the conditions by areas interaction allowed the classification of areas into groups for each maintenance condition. Areas were grouped by

Table 1. Strategies used by the subjects during the retention stage under the point-light and video maintenance conditions

Point-light maintenance condition Video maintenance condition

$\begin{array}{llr}\text { Subvocal rehearsal } & 7 & 6 \\ \text { Mental imagery + subvocal rehearsal } & 4 & 5 \\ \text { No strategies } & 1 & 1\end{array}$

Table 2. Summary of the 2 (conditions) 6 (areas) repeated-measures ANOVAs for each frequency band

\begin{tabular}{|c|c|c|c|c|c|c|c|c|c|c|}
\hline & \multicolumn{2}{|c|}{$4-8 \mathrm{~Hz}$} & \multicolumn{2}{|c|}{$8-10 \mathrm{~Hz}$} & \multicolumn{2}{|c|}{$10-13 \mathrm{~Hz}$} & \multicolumn{2}{|c|}{$13-20 \mathrm{~Hz}$} & \multicolumn{2}{|c|}{$20-30 \mathrm{~Hz}$} \\
\hline & $\mathrm{F}$ & $\mathrm{P}$ & $\mathrm{F}$ & $\mathrm{P}$ & $\mathrm{F}$ & $\mathrm{P}$ & $\mathrm{F}$ & $\mathrm{P}$ & $\mathrm{F}$ & $\mathrm{P}$ \\
\hline Condition & 1.169 & .30 & 35.278 & * & 2.411 & .15 & 0.478 & .50 & 2.030 & .18 \\
\hline Area & 0.335 & .89 & 0.974 & .44 & 0.825 & .54 & 0.707 & .62 & 1.028 & .41 \\
\hline Condition area & 0.246 & .94 & 0.683 & .64 & 1.234 & .31 & 2.624 & $*$ & 1.892 & .11 \\
\hline
\end{tabular}

${ }^{*}$ Indicates statistically significant difference $(\mathrm{P}<.05)$.

similar PLI final $($ see Fig. 4). This way of proceeding allows a quick and clear view of significant differences between areas. For example, in the $13-20 \mathrm{~Hz}$ frequency band under the point-light motion maintenance condition, $\mathrm{PLI}_{\text {final }}$ for the frontal area, the left temporal area, the central area, the right temporal area, the parietal area, and the occipital area were 1.01072, 0.99565, $1.06177,1.02180,1.06552,1.01129$ respectively. These areas can be ranked in the following decreasing order: parietal area, central area, right temporal area, occipital area, frontal area, and left temporal area. Duncan post hoc test revealed that the parietal area was not statistically different to the central area whereas the central area was statistically different to the right temporal, occipital, frontal and left temporal areas. The right temporal area was not statistically different to the occipital, frontal, and left temporal areas. Two groupings were thus made according to the $\mathrm{PLI}_{\text {final }}$ values' statistical equality under the point-light motion maintenance condition (see Fig. 4). Using the same approach, under the video motion maintenance, three groups of areas were identified (see Fig. 4).

\section{DISCUSSION}

The aim of this study was to investigate the mechanisms to maintain in memory, for short periods of time, familiar movement information stemming on one hand from an unfamiliar display and on the other hand from a realistic, familiar display. As expected, results showed that these mechanisms were different in the beta frequency band. First, functional connectivity, assessed by PLI, differed within the frontal and right temporal areas under the two maintenance conditions (i.e., point-light and video maintenance conditions). Second, functional connectivity between areas displayed different patterns under the maintenance conditions. To explain these findings, the discussion has been organized into four sections. The first section discusses qualitative data while the second considers EEG activity data. The third focuses

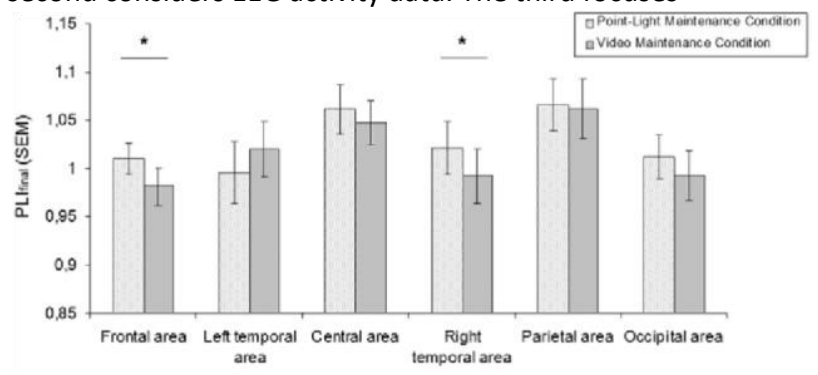

Fig. 3. PLlfinal values in the $13-20 \mathrm{~Hz}$ frequency band under the point-light and video motion maintenance conditions within the frontal, temporal, central, parietal, and occipital areas. Asterisk () indicates statistically significant difference $(\mathrm{P}<.05)$.

on functional connectivity within the frontal and right temporal areas and the last reviews functional connectivity between areas.

The reader should bear in mind that caution must be exerted when interpreting the results of this study. Stimuli presented to the subjects (i.e., biological movements) were different to those reported in conventional working memory studies (i.e., letters, digits, words, smooth shapes, matrixes containing coloured targets, and spatial locations). Consequently, the durations of the cue stimulus and retention stages are much longer than those employed in traditional studies of working memory. In traditional studies, durations are about a subsecond for the presentation of the cue stimulus and a few seconds for the retention stage. This disparity does not allow for absolute comparisons since different mechanisms may underlie the working memory maintenance process in these two cases.

\section{Qualitative data}

Qualitative data stemming from the interviews fit the theoretical model of working memory devised by Baddeley and Hitch 
(1974), Baddeley $(1986,2000,2010)$ and Repovs and Baddeley (2006). During the

Point-light maintenance condition

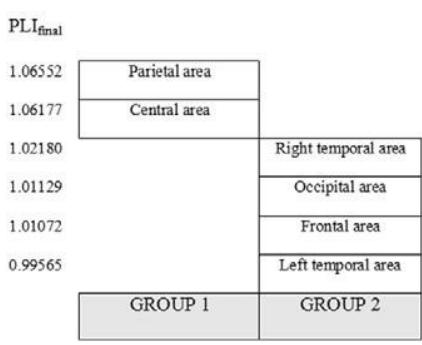

agreement with previous data which have reported particular activity in beta frequency band when nonhuman primates and Video maintenance condition

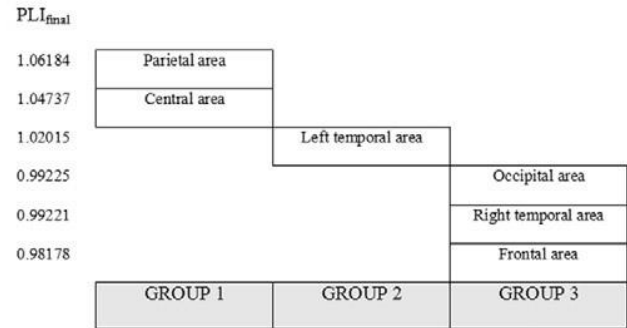

Fig. 4. Area classification in groups in the $13-20 \mathrm{~Hz}$ frequency band for each maintenance condition. This classification allows a quick and clear

view of significant differences between areas.

retention stage, the subjects reported using either subvocal rehearsal or a combination of subvocal rehearsal and imagery to help them retain movement information in order to be able to recognize them at a later stage. These strategies are rehearsal strategies which prevent the information from decaying. This can correspond to the process of active rehearsal described by Baddeley $(1986,2000,2010)$. Two subjects also declared using no strategies. This can reflect passive storage of information which is one component of the maintenance mechanism in Baddeley's working memory model. This can also mean that these two subjects were not aware of the strategies they employed. Implicit strategies have been used. Finally, most of the subjects declared using subvocal rehearsal alone or in combination with mental imagery. This point reveals that the subjects transformed the visually-presented movements into subvocal representations and repeated them during the retention stage. They recoded visual items into phonological forms. This strategy can be linked to the phonological loop model (Baddeley, 1986; Burgess and Hitch, 1999). The phonological loop can deal with information that is presented visually. This visual information is transformed into a phonological code by subvocalization (Wilson and Emmorey, 1997) and is thus able to enter the phonological store. This process can be designated as a manipulation process which has been characterized by Henson (2001, p. 151) as involving more than a simple maintenance,

i.e. it requires a transformation or "re-representation." For example, reordering the words of a presented list in alphabetic order could be defined as a manipulation.

\section{EEG activity data}

The presence of functional connectivity differences in the beta frequency band (i.e., $13-20 \mathrm{~Hz}$ ) was expected and is in accordance with the results of earlier studies (Tallon-Baudry et al., 1998, 1999, 2001; Hwang et al., 2005; Leiberg et al., 2006; Pesonen et al., 2007; Altamura et al., 2010; Calmels et al., 2011). These studies have shown that beta oscillations were related to the rehearsing process of the sensory trace in memory. This point is in accordance with the present subjects' reports. The majority of them declared using subvocal rehearsal and (mental) imagery to help them maintain in memory movement information. This difference in beta frequency band is also in humans anticipated the presentation of visual stimuli (Gross et al., 2004, 2006; Zhang et al., 2008; Altamura et al., 2010): a synchronization activity in the beta frequency band was observed. For instance, Altamura et al. (2010), using MEG, investigated MEG signal modulations when subjects performed a modified version of a letter Sternberg memory task. During the preparatory stage, increases in beta power (i.e., synchronization) within the dorsolateral prefrontal cortex, the left inferior prefrontal gyrus, and the left parietal and temporal areas were revealed. During the retention stage, a decrease in beta power (i.e., desynchronization) within the prefrontal areas and an increase in beta power within the visual areas were also detected. In the present study, the subjects knew in advance which kind of display would be presented in the recognition task. Indeed, the display configuration in the observed stage (i.e., stage 2) was similar to the one shown during the recognition task stage (i.e., stage 4). For example, if in stage 2 , a video display was exhibited, a video display would have been presented in stage 4 . The same principle has been applied for a point-light display. It is therefore likely that the subjects anticipated the forthcoming task and that the beta rhythm responses were associated to the anticipatory processing.

Functional connectivity PLI differences were detected within the frontal and (right) temporal areas. Interestingly, these two areas are recognized as playing a role during the delay period of a working memory task

(i.e., retention period) (Smith et al., 1995; Barch et al., 1997; Courtney et al., 1997; Druzgal and d'Esposito, 2003; Ranganath et al., 2004; Sakai and Passingham, 2006; Altamura et al., 2010). For example, during the working memory delay period, Ranganath et al. (2004) with fMRI have shown the involvement of the prefrontal and inferior temporal cortices which have complementary roles. The temporal cortex was more sensitive to the type of information that had to be retained in memory whereas the prefrontal cortex was affected by memory load.

Computation of PLI may be slightly affected by the signal-tonoise ratio. There may be a possible effect of difference in amplitude on signal-to-noise ratio which can impinge upon phase measurement and indirectly upon PLI. However, in the present study, a lot of noise (e.g., amplifier, electrode noise, muscle activity) was outside the pass band of the data. Besides, ocular artefacts were corrected and a ratio procedure was employed. PLI values were also averaged across trials for each subject permitting improved signal-to-noise ratio. These points lead to the conclusion that signal-to-noise ratio is not a typical 
problem of PLI and does not likely play a very large role in this study.

Finally, maintaining motion information presented via video displays resulted in less functional connectivity than passively staring at an amber screen within the frontal, right temporal, and occipital areas. This finding suggests that functional connectivity is more extensive during a resting state than during an active state. This point is supported by Shulman et al. (1997), Mazoyer et al. (2001), and Wicker et al. (2003). In their metaanalysis, these authors have shown that groups of brain areas were active at rest (i.e., in a state were the eyes are closed, during visual fixation, during passive observation of a visual stimuli, or in situations in which relaxing and thinking of nothing was proposed) and that this activity decreased during the completion of diversified goaldirected actions. In the literature, this process refers to the concept of 'default mode.' It has been revealed mainly via $\mathrm{fMRI}$ or positron emission tomography (PET) techniques and was composed of a network including the medial temporal lobe, the medial, lateral, and inferior parietal regions, the precuneus, and the medial prefrontal and post cingulate cortices. A question that may arise is why the same phenomenon was not observed under the maintenance of motion information presented through point-light displays. It can be speculated that maintenance under that condition was more demanding than maintenance under the video condition. This suggestion corroborates the subjects' report. Subjects perceived the maintenance and recognition of movements from a point-light display as more difficult than the maintenance and recognition from a video display. This perception is in agreement with the scores obtained by the subjects on the Likert scale and the percentages of correct answers when performing the Sternberg task under the two maintenance conditions. The subjects also declared themselves to be more concentrated and focused on details during the retention period under the pointlight motion condition. Perception of difficulty under the pointlight condition may have required more energy compared to that needed under the video and control conditions. This cost analysis implies that maintaining motion information presented via point-light displays may have resulted in more functional connectivity than passively observing a monochrome amber screen.

\section{Functional connectivity within the frontal and right temporal} areas

Functional connectivity within the frontal and right temporal areas was greater under the point-light motion maintenance condition compared to the video motion maintenance condition. It can be suggested that cortical activity during the retention stage is sensitive to the expected perceptual difficulty of the upcoming task to perform (i.e., recognition task). In the present study, the subjects knew in advance which kind of display would be presented in the recognition task. It is therefore possible that the subjects anticipated the forthcoming task. They had expectations on the demands, on the perceptual difficulty of this task. Under the point-light motion maintenance condition, the higher values of functional connectivity within the frontal and right temporal areas could be the result of a greater cognitive demand, a greater attentional demand which may have a cost and can recruit additional neuron population. This explanation corroborates the subjects' report (see above) and concurs with the findings of Offen et al. (2009), Ress et al. (2000), and Lepsien et al. (2011). Ress et al. (2000) reporting that preparatory cortical activity increased as task difficulty increased. Along the same lines, work by Offen et al. (2009) and Lepsien et al. (2011) has shown that during working memory maintenance, activity in the visual cortex intensified as a function of the expected difficulty of an upcoming recognition task. Following this line of reasoning, it can be suggested that the anticipation explanation is to some degree related to the process of attention. As reported by Reuter-Lorenz and Jonides (2008), all working memory tasks entail attentional control but the degree of this control is modulated by task demands, individual differences, and/or ageing. In the present case, task demands seem to be the cause of this modulation. Expectation of the perceptual difficulty of the upcoming task could have generated a greater attentional demand which was expressed by a higher functional connectivity under the point-light motion maintenance condition.

A second explanation derived from the subjects' detailed debriefs, can be proposed. This explanation is centred on the strategy of mental imagery. While admittedly speculative because of the unequal distribution between the reported strategies used by the subjects, the authors made the decision to briefly evoke this explanation. While it is potentially an interesting suggestion, additional investigation is required for its support. Here, subjects stated that mental imagery used in the retention stage, after the exposure of movements embedded in impoverished and unfamiliar displays which are devoid of colour and form information, took into account the colour, the shape, the shading, and the contours of movements. This means that subjects had to code the visual information presented through Johansson's point light patterns into a mental representation including structural and contextual information such as colours, forms, and contours. This mental rearrangement or manipulation may entail additional resources which could be expressed by a higher functional connectivity within the frontal and right temporal areas under the point-light motion maintenance condition compared to the video motion maintenance condition. This interpretation is in accordance with findings presented by Sauseng et al. (2005) who observed stronger cortical coupling as subjects had to manipulate spatial information and to maintain it in memory compared to the situation in which they had only to keep this information in memory before completing a recognition task.

\section{Functional connectivity between areas}

Under the point-light maintenance condition, functional connectivity was greater within the parietal and central areas compared to the four other areas. Under the video maintenance condition, a different pattern was seen. Functional connectivity of the left temporal area was weaker compared to those of parietal and central areas and stronger than those of occipital, right temporal and frontal areas. It means that functional connectivity between areas changes according to the working memory task performed and more specifically to the display in which the movement to retain was presented. The left temporal 
area seems to play a specific role. This result is difficult to explain. What can be advanced is that activity within the left temporal area may indirectly reflect the activity of Wernicke's area, an area only present in the left hemisphere and considered to be devoted to phonological storage (Paulesu et al., 1993). It can thus be speculated that under the video maintenance condition, the subjects may have verbally coded and stored in Wernicke's area, additionally to motion information, information related to colour and form. This greater amount of colour and form information might have required additional cortical resources which could have been expressed by a higher functional connectivity within the left temporal area compared to that detected within the right temporal, occipital and frontal areas under the video maintenance condition. In contrast to the video display, the point-light display is a minimalist display devoid of colour and form information. The storage of information stemming from this kind of display thus required less resources, less cognitive demands possibly leading to a lack of significant differences in functional connectivity between the left temporal area and the right temporal, occipital and frontal areas. This interpretation seems to be in line with the trend observed within the left temporal area when $\mathrm{PLI}_{\text {final }}$ value under the point-light maintenance condition was compared with that detected in the video maintenance condition (0.99565 vs 1.02015, $\mathrm{P}=.07$ ).

\section{CONCLUSION}

In conclusion, the study reveals that the mechanisms to maintain temporarily in memory familiar movement information stemming from an unfamiliar display are different to those involved when (movement) information emanates from a familiar display. Changes in functional connectivity were mainly discussed in the light of the process of anticipation. Subjects' perception of the expected difficulty of the upcoming recognition task has been considered. However, further experiences are needed to cast some light on working memory maintenance process of biological movements.

Acknowledgements-This study was supported by a grant from the French Ministry of Health, Youth, and Sports. The funders had no role in study design, data collection, analysis and interpretation, decision to publish, or preparation of the manuscript.

The authors are grateful to the participants who took part in this study and to Magaly and Me'lany Hars for the stimuli construction, the video production, and the video footage editing. Part of this paper was presented at the 18th Annual Meeting of the Organization for Human Brain Mapping, Beijing, June 2012.

\section{REFERENCES}

Altamura M, Goldberg TE, Elvevag B, Holroyd T, Carver FW, Weinberger DR, Coppola R (2010) Prefrontal cortex modulation during anticipation of working memory demands as revealed by magnetoencephalography. Int J Biomed Imaging 2010. http:// dx.doi.org/10.1155/2010/840416.

Baddeley AD Working memory. Oxford: University Press; 1986.

Baddeley AD (2000) The episodic buffer: a new component of working memory? Trends Cogn Sci 4:417-423.
Baddeley AD Working memory thought, and action. Oxford: University Press; 2010.

Baddeley AD, Hitch G Working memory. In: Bower GH, editor. The psychology of learning and motivation: advances in research and theory. New York: Academic Press; 1974. p. 47-89.

Barch DM, Braver TS, Nystrom LE, Forman SD, Noll DC, Cohen JD (1997) Dissociating working memory from task difficulty in human prefrontal cortex. Neuropsychologia 35:1373-1380.

Burgess N, Hitch GJ (1999) Memory for serial order: a network model of the phonological loop and its timing. Psychol Rev 106:551-581.

Calmels C, Foutren M, Stam CJ (2011) Influences of instructions and expertise on the mechanisms involved during a working memory task. J Psychol 25:105-115.

Calmels C, Holmes P, Jarry G, Hars M, Lopez E, Paillard A, Stam C (2006) Variability of EEG synchronization prior to and during observation and execution of a sequential finger movement. Hum Brain Mapp 27:251266.

Cochin S, Barthelemy C, Lejeune B, Roux S, Martineau J (1998) Perception of motion and qEEG activity in human adults. Electroenceph Clin Neurophysiol 107:287-295.

Courtney SM, Ungerleider LG, Keil K, Haxby JV (1997) Transient and sustained activity in a distributed neural system for human working memory. J Psychol 386:608-611.

Douw L, van Dellen E, de Groot M, Heimans JJ, Klein M, Stam CJ, Reijneveld JC (2010) Epilepsy is related to theta band brain connectivity and network topology in brain tumor patients. BMC Neurosci 23:11-103.

Druzgal TJ, d'Esposito M (2003) Dissecting contributions of prefrontal cortex and fusiform face area to face working memory. J Cogn Neurosci 15:771784.

Fiehler K, Burke M, Engel A, Bien S, Rosler F (2008) Kinesthetic working memory and action control within the dorsal stream. Cereb Cortex 18:243-253.

Gross J, Schmitz F, Schnitzler I, Kessler K, Shapiro K, Hommel B, Schnitzler A (2004) Modulation of long-range neural synchrony reflects temporal limitations of visual attention in humans. Proc Natl Acad Sci USA 101:13050-13055.

Gross J, Schmitz F, Schnitzler I, Kessler K, Shapiro K, Hommel B, Schnitzler A (2006) Anticipatory control of long-range phase synchronization. Eur J Neurosci 24:2057-2060.

Hars M, Hars M, Stam C, Calmels C (2011) Effects of visual context upon functional connectivity during observation of biological motions. PLoS ONE 6(10):e25903.

Henson RNA Neuronal working memory: applications of the working memory model to neuropsychology and neuroimaging. In: Andrade J, editor. Working memory: a work in progress. London: Routeledge; 2001. p. 151-173. Hwang G, Jacobs J, Geller A, Danker J, Sekuler R, Kahana MJ (2005) EEG correlates of verbal and nonverbal working memory. Behav Brain Funct 1:20.

Johansson G (1973) Visual perception of biological motion and a model for its analysis. Percept Psychophys 14:201-211.

Lepsien J, Thornton I, Nobre A (2011) Modulation of working-memory maintenance by directed attention. Neuropsychologia 49: 1569-1577.

Leiberg S, Lutzenberger W, Kaiser J (2006) Effects of memory on cortical oscillatory activity during auditory pattern working memory. Brain Res 1120:131-140.

Long BC, van Stavel R (1995) Effects of exercise training on anxiety: a metaanalysis. J Appl Sport Psychol 7:167-189.

Mazoyer B, Zago L, Mellet E, Bricogne S, Etard O, Houde' O, Crivello F, Joliot M, Petit L, Tzourio-Mazoyer N (2001) Cortical networks for working memory and executive functions sustain the conscious resting state in man. Brain Res Bull 54:287-298.

McFarland DJ, Miner LA, Vaughan TM, Wolpaw JR (2000) Mu and beta rhythm topographies during motor imagery and actual movements. Brain Topogr 12:177-186

Neuper C, Pfurtscheller G Motor imagery and ERD. In: Pfurtscheller G, Lopes da Silva FH, editors. Event-related desynchronization. Handbook of electroencephalography and clinical neurophysiology. Amsterdam: Elsevier; 1999. p. 303-325. 
Nunez A, Amzica F, Steriade M (1992) Intrinsic and synaptically generated delta $(1-4 \mathrm{~Hz})$ rhythms in dorsal lateral geniculate neurons and their modulation by light-induced fast $(30-70 \mathrm{~Hz})$ events. Neuroscience 51:269-284.

Nuwer MR, Lehmann FL, da Silva D, Matsuoka S, Sutherling W, Vibert JF (1999) IFCN guidelines for topographic and frequency analysis of EEGs and EPs. The International Federation of Clinical Neurophysiology. Electroencephalog Clin Neurophysiol Suppl 52:15-20.

Offen S, Schluppeck D, Heeger DJ (2009) The role of early visual cortex in visual short-term memory and visual attention. Vision Res 49:1352-1362.

Oldfield RC (1971) The assessment and analysis of handedness: the Edinburgh inventory. Neuropsychologia 9:97-113.

Paulesu E, Frith CD, Frackowiak RS (1993) The neural correlates of the verbal component of working memory. Nature 362:342-345.

Pesonen M, Hamalainen $\mathrm{H}$, Krause CM (2007) Brain oscillatory 4-30Hz responses during a visual $n$-back memory task with varying memory load. Brain Res 1138:171-177.

Pfurstcheller G, Neuper C (1997) Motor activity activates primary sensorimotor area in humans. Neurosci Lett 239:65-68.

Pfurtcheller G, Neuper C, Flotzinger D, Pregenzer M (1997) EEGbased discrimination between imagination of right and left hand movement. Electroen Clin Neuro 103:1-10.

Pineda JA, Hecht E (2009) Mirroring and mu rhythm involvement in social cognition: are there dissociable subcomponents of theory of mind? Biol Psychol 80:306-314.

Pochon JB, Levy R, Poline JB, Crozier S, Le' he' ricy S, Pillon B, Deweer B, Le Bihan D, Dubois B (2001) The role of dorsolateral prefrontal cortex in the preparation of forthcoming actions: an fMRI study. Cereb Cortex 11:260266.

Postle BR, Berger JS, Taich AM, d'Esposito M (2000) Activity in human frontal cortex associated with spatial working memory and saccadic behavior. J Cogn Neurosci 12:2-14.

Ress D, Backus BT, Heeger DJ (2000) Activity in primary visual cortex predicts performance in a visual detection task. Nat Neurosci 3:940-945.

Ranganath C, DeGustis J, d'Esposito M (2004) Category-specific modulation of inferior temporal activity during working memory encoding and maintenance. Cogn Brain Res 20:37-45.

Repovs G, Baddeley A (2006) The multi-component model of working memory: explorations in experimental cognitive psychology. Neuroscience 139:5-21.

Reuter-Lorenz PA, Jonides J The executive is central to working memory. In: Conway ARA, Jarrold C, Kane MJ, Miyake A, Towse JN, editors. Insights from age performance and task variations.

Variation in working memory. New York: Oxford University Press; 2008. p. 250-271.

Sakai K, Rowe JB, Passingham RE (2002) Active maintenance in prefrontal area 46 creates distractor-resistant memory. Nat Neurosci 5:479-484.
Sakai K, Passingham RE (2006) Prefrontal set activity predicts rulespecific neural activity during subsequent cognitive performance. J Neurosci 26:1211-1218.

Sarthein J, Petsche H, Rappelsberger P, Shaw GL, Von Stein A (1998) Synchronization between prefrontal and posterior association cortex during human working memory. Proc Natl Acad Sci USA 95:7092-7096.

Sauseng P, Klimesch W, Doppelmayr M, Pecherstorfer T, Freunberger R, Hanslmayr S (2005) EEG alpha synchronization and functional coupling during top-down processing in a working memory task. Hum Brain Mapp 26:148-157.

Shulman GL, Fiez JA, Corbetta M, Buckner RL, Miezen F, Raichle ME, Petersen SE (1997) Common blood changes across visual tasks. II: Decreases in cerebral cortex. J Cogn Neurosci 9:648-663.

Siegel S Non parametric statistics for the behavioural sciences. New York: McGraw-Hill, Inc.; 1956.

Smith EJ, Balogh A, Burton ME, Erdos G, Forsyth RJ (1995) Results of the Ulysses fast latitude scan: magnetic field observations. Geophys Res Lett 22:3325.

Stam CJ, de Bruin EA (2004) Scale-free dynamics of global functional connectivity in the human brain. Hum Brain Mapp 22:97-109.

Stam CJ, Van Cappellen van Walsum AM, Micheloyannis S (2002) Variability of EEG synchronization during a working memory task in healthy subjects. Int J Psychophysiol 46:53-66.

Stam CJ, Nolte G, Daffertshofer A (2007) Phase lag index: assessment of functional connectivity from multi channel EEG and MEG with diminished bias from common sources. Hum Brain Mapp 28:1178-1193.

Tallon-Baudry C, Bertrand O, Peronnet F, Pernier J (1998) Induced cband activity during the delay of a visual short-term memory task in humans. J Neurosci 18:4244-4254.

Tallon-Baudry C, Kreiter A, Bertrand O (1999) Sustained and transient oscillatory responses in the gamma and beta bands in a visual short-term memory task in humans. Vis Neurosci 16:449-459.

Tallon-Baudry C, Bertrand O, Fischer C (2001) Oscillatory synchrony between human extrastriate areas during visual short-term memory maintenance. J Neurosci 21:1-5.

Ulloa ER, Pineda JA (2007) Recognition of point-light biological motion: mu rhythms and mirror neuron activity. Behav Brain Res 183:188-194.

Vermersch P L'entretien d'explicitation. Paris: Edition ESF; 2003.

Wicker B, Ruby P, Royet JP, Fonlupt P (2003) A relation between rest and the self in the brain? Brain Res Rev 43:224-230.

Wilson M, Emmorey M (1997) A visuospatial «phonological loop» in working memory: evidence from American sign language. Mem Cognit 25:313320.

Zhang Y, Wang X, Bressler SL, Chen Y, Ding M (2008) Prestimulus cortical activity is correlated with speed of visuomotor processing. J Cogn Neurosci 20:1915-1925. 